\title{
Wiener-Hopf analysis of an M/G/1 queue with negative customers and of a related class of random walks*
}

\author{
N. Bayer and O.J. Boxma \\ CWI, P.O. Box 94079, 1090 GB Amsterdam, The Netherlands
}

Received 5 October 1995; revised 12 July 1996

\begin{abstract}
Two variants of an M/G/1 queue with negative customers lead to the study of a random walk $\boldsymbol{X}_{n+1}=\left[\boldsymbol{X}_{n}+\boldsymbol{\xi}_{n}\right]^{+}$where the integer-valued $\boldsymbol{\xi}_{n}$ are not bounded from below or from above, and are distributed differently in the interior of the state-space and on the boundary. Their generating functions are assumed to be rational. We give a simple closed-form formula for $\mathbb{E}\left(s^{X_{n}}\right)$, corresponding to a representation of the data which is suitable for the queueing model. Alternative representations and derivations are discussed. With this formula, we calculate the queue length generating function of an $\mathrm{M} / \mathrm{G} / \mathrm{l}$ queue with negative customers, in which the negative customers can remove ordinary customers only at the end of a service. If the service is exponential, the arbitrarytime queue length distribution is a mixture of two geometrical distributions.
\end{abstract}

Keywords: M/G/1 queue, negative customers, queue length, Wiener-Hopf technique.

\section{Introduction}

Consider the following model ${ }^{1}$. Independent jobs arrive at a server in a Poissonian stream. At the same time, the server also admits a Poissonian stream of messages from other service sites, inviting jobs to immigrate there. The server handles these messages during a housekeeping routine, which is re-entered after every job completion. Single jobs are matched with single messages, and sent to the sites where the messages had originated. Surplus messages are discarded. The performance question is: How many jobs are found at the server at an arbitrary time? In this model, the messages can be seen as negative customers, who each remove an ordinary customer at the end of a service.

We show that such a problem is fully tractable, assuming that the LaplaceStieltjes transform of the service distribution is rational. Apart from analyzing a

\footnotetext{
* Supported by the European grant BRA-QMIPS of CEC DG XIII.

${ }^{1}$ It may be interpreted as a rudimentary depiction of a load balancing mechanism, but is given here merely for the sake of illustration.
}

(C) J.C. Baltzer AG, Science Publishers 
specific generalization of the classical M/G/1 queue, this paper also makes a contribution to the theory of one-dimensional random walks. Indeed, in fact we study a rather general class of random walks, a special case of which describes the evolution of the number of jobs after service completions in the above queueing model. These integer-valued random walks have unbounded jumps upward and downward; they are truncated at zero, and have a different step size distribution at the boundary. We do not assume any special structure of the step size, e.g., a difference between two nonnegative variates with known distributions. We mostly assume that the step size generating functions are rational, which allows us to obtain very explicit results for the steady-state distribution of the random walk. The random-walk analysis is based on Wiener-Hopf theory. In principle the Wiener-Hopf theory allows a solution of the problem without taking recourse to rationality assumptions, but such assumptions have the very attractive feature of yielding much more explicit solutions while not posing serious restrictions from a modeling point of view.

After having solved the random walk problem, we specify the results for the above-mentioned queueing model. Having found its embedded-time queue-length generating function, we exploit the piecewise Poissonian nature of the process to derive the steady-state queue-length generating function. Interestingly, this conversion alone produces an immediate alternative proof of the classical Pollaczek-Khintchine formula for a conventional $M / G / 1$ queue.

\subsection{Related literature}

See Cohen [6] for an introductory exposition of the application of the WienerHopf technique (with bivariate transforms) to various types of problems. Further expositions on the subject, in relation to random walks and queues, are contained in the books of Asmussen [1], Borovkov [3], Cohen [7], and Kleinrock [11]. More bibliography, concerning further aspects of the Wiener-Hopf technique, is included in Bayer [2]. An extensive discussion of the analysis of two-dimensional random walks in the first quadrant, and their solution via the theory of boundary value problems (Wiener-Hopf and similar problems: Dirichlet, Riemann, Riemann-Hilbert) is contained in Cohen and Boxma [8].

The invitation messages in the example at the beginning of this section act as "negative customers". Queueing systems with negative customers were recently introduced by Gelenbe [9], and presently attract much attention. In contrast with the ordinary customers, negative customers are not served but a negative customer removes one ordinary customer from the queue. Negative customers have been given interpretations, for example, as work removal signals in production networks, or as inhibitor signals in neural networks. In the literature on negative customers it has so far been assumed that a negative customer upon its arrival immediately removes an ordinary customer; our assumption of customer removal at service completion epochs (see the above-described model) seems rather natural in some of the above-mentioned application areas, as well as in cases where the server can inspect customers only at service completion epochs to see whether they still need to be served. 
For the $\mathrm{M} / \mathrm{G} / 1$ queue with negative customers who upon their arrival immediately remove an ordinary customer, the queue-length distribution is analysed by Harrison and Pitel [10]. Their analysis of the generating function for the equilibrium queue-length distribution eventually leads to a Fredholm integral equation of the first kind, that must be solved numerically - which is a notoriously difficult problem. Boucherie and Boxma [4] analyse a closely related model that is amenable to a detailed exact analysis: an M/G/1 queue with negative customers, in which a negative customer removes a random amount of work that does not necessarily correspond to an integer number of customers. Their analysis leads to a Wiener-Hopf equation for the Laplace-Stieltjes transform of the equilibrium distribution of the workload in the queue; it can be solved.

\subsection{Organization of the paper}

We begin in section 2 with a formal introduction of the class of random walks under investigation, and with stating a result regarding their stationary generating function. The particular representation of this result suits our $M / G / 1$ queue with negative customers. Two variants of this queue are formally introduced in section 3 ; simple procedures for computing the embedded-time queue-length generating functions are given, using the result of section 2 . The conversion to arbitrary time is given in section 4 . Section 5 is dedicated to the Wiener-Hopf proof of the random walk result, and to a discussion of alternative assumptions, representations, and derivations.

\section{Description and result for the random walk}

\subsection{Description of the random walk} equation $^{2}$

We study a random walk $\left\{\boldsymbol{X}_{n}\right\}$ on the nonnegative integers, with the evolution

$$
\boldsymbol{X}_{n+1}=\left[\boldsymbol{X}_{n}+\boldsymbol{\xi}_{n}\right]^{+} .
$$

The driving sequence $\left\{\boldsymbol{\xi}_{n}\right\}$ assumes integer values, which are not necessarily bounded from below or from above. $\boldsymbol{\xi}_{n}$ depends on the history $\left\{\boldsymbol{X}_{j}\right\}_{j \leqslant n}$ only through the event $\left\{\boldsymbol{X}_{n}>0\right\}$. It is convenient to suppose that $\boldsymbol{\xi}_{n}$ is selected from one of two independent i.i.d. streams, $\left\{\boldsymbol{\xi}_{n}^{\text {interior }}\right\}$ and $\left\{\boldsymbol{\xi}_{n}^{\text {boundary }}\right\}$, through the rule

$$
\boldsymbol{\xi}_{n}= \begin{cases}\boldsymbol{\xi}_{n}^{\text {interior }} & \text { if } \boldsymbol{X}_{n}>0 \\ \boldsymbol{\xi}_{n}^{\text {boundary }} & \text { if } \boldsymbol{X}_{n}=0\end{cases}
$$

${ }^{2}[x]^{+}$and $[x]^{-}$stand for $\max (0, x)$ and $\min (0, x)$, respectively. 
The random walk is thus specified by the two generating functions

$$
\begin{aligned}
A(s) \triangleq \mathbb{E}\left(s^{\xi_{n}} \mid X_{n}>0\right)=\mathbb{E}\left(s^{\xi_{n}^{\text {miterior }}}\right), & |s|=1, \\
A_{0}(s) \triangleq \mathbb{E}\left(s^{\xi_{n}} \mid \boldsymbol{X}_{n}=0\right)=\mathbb{E}\left(s^{\xi_{n}^{\text {boundary }}}\right), & |s|=1,
\end{aligned}
$$

which are both assumed to be rational. $\boldsymbol{\xi}_{n}^{\text {interior }}$ is assumed to be aperiodic, and must satisfy the stability condition

$$
\mathbb{E}\left(\xi_{n}^{\text {interior }}\right)<0 .
$$

Our quantity of interest is the generating function

$$
F(s) \triangleq \mathbb{E}\left(s^{X_{n}}\right), \quad|s| \leqslant 1 .
$$

The representation of $A(s)$ and of $A_{0}(s)$ is of relevance to the derivation and the representation of the solution. $A(s)-1$ has a reduced rational representation (i.e., without zeros common to both numerator and denominator) of the form

$$
A(s)-1=C \frac{\prod_{i=1}^{k}\left(s-\alpha_{i}\right)}{\prod_{j=1}^{n}\left(s-\beta_{j}\right)}(s-1),
$$

where none of the $\alpha_{1}, \ldots, \alpha_{k}$ or the $\beta_{1}, \ldots, \beta_{n}$ are on the unit circle, and

$$
\left|\left\{i /\left|\alpha_{i}\right|<1\right\}\right|-\left|\left\{j /\left|\beta_{j}\right|<1\right\}\right|=-1 ;
$$

we refer to the companion paper [2] for a derivation of equation (4), and of the following factorization of $A(s)-1$, which forms the basis for the determination of $F(s)$ :

$$
A(s)-1=C \cdot(s-1) \frac{H_{\text {in }}(s)}{H_{\text {out }}(s)}, \quad|s|=1,
$$

where

$$
H_{\text {in }}(s) \triangleq \frac{\prod_{\left\{i /\left|\alpha_{i}\right|>1\right\}}\left(s-\alpha_{i}\right)}{\prod_{\left\{j /\left|\beta_{j}\right|>1\right\}}\left(s-\beta_{j}\right)}, \quad|s| \leqslant 1,
$$

and

$$
H_{\text {out }}(s) \triangleq \frac{\prod_{\left\{j /\left|\beta_{j}\right|<1\right\}}\left(s-\beta_{j}\right)}{\prod_{\left\{i /\left|\alpha_{i}\right|<1\right\}}\left(s-\alpha_{i}\right)}, \quad|s| \geqslant 1 .
$$

The factorization is chosen such that $H_{\text {in }}(s)$ is analytic in $|s| \leqslant 1$ and $H_{\text {out }}(s)$ is analytic in $|s| \geqslant 1$. We incorporate the description of $A_{0}(s)$ in a representation of $A_{0}(s) / A(s)-1$. Such a representation is natural when a relation of the type

$$
\boldsymbol{\xi}_{n}^{\text {boundary }} \stackrel{D}{=} \boldsymbol{\xi}_{n}^{\text {interior }}+\boldsymbol{\delta}_{n}
$$


where $\stackrel{D}{=}$ denotes equality in distribution, is known to hold, and $\boldsymbol{\delta}_{n}$ is independent of $\xi_{n}^{\text {interior }}$ and has a known generating function (examples occur in various M/G/1-type queueing models). Alternative representations are discussed in subsection 5.2. Let then

$$
\frac{A_{0}(s)}{A(s)}-1=(s-1) \frac{U(s)}{V(s)}, \quad|s|=1,
$$

be a reduced rational representation. We may assume that the polynomials $U(s)$ and $V(s)$ are of the form

$$
\begin{aligned}
& U(s)=\sum_{j=0}^{\ell} u_{j} s^{j} \\
& V(s)=\sum_{j=0}^{m} v_{j} s^{j}=\prod_{j=1}^{m}\left(s-\omega_{j}\right)
\end{aligned}
$$

we tacitly assumed that $v_{m}=1$. Clearly, none of the $\omega_{1}, \ldots, \omega_{m}$ can be equal to 1 . We assume, without loss of generality, that $\omega_{1}, \ldots, \omega_{q}$ are in $|s| \leqslant 1$ while $\omega_{q+1}, \ldots, \omega_{m}$ are in $|s|>1$, for some $0 \leqslant q \leqslant m$.

\subsection{Statement of the result}

Theorem 1. If the poles of $A_{0}(s) / A(s)$ (namely $\omega_{1}, \ldots, \omega_{m}$ ) are distinct from each other and from zero then

$$
F(s)=\frac{V(1)}{V(s)} \cdot \frac{H_{\text {in }}(1)}{\overrightarrow{1} M^{-1} \vec{\vartheta}}\left[\frac{\vec{s} M^{-1} \vec{\vartheta}}{H_{\text {in }}(s)}-(s-1) U(s)\right], \quad|s| \leqslant 1,
$$

where the matrix $M$ and vector $\vec{\vartheta}$ are defined hereafter, $\overrightarrow{1}$ is a vector of all ones, and $\vec{s}$ is a vector of powers of $s$, starting from the zero'th power.

The proof is deferred to section 5. The treatment of the case where the hypothesis concerning the $\omega_{1}, \ldots, \omega_{m}$ fails to hold is discussed in subsection 5.2.

Remark 2.1 (two important special cases).

Case 1. $\xi_{n}^{\text {boundary }} \stackrel{D}{=} \boldsymbol{\xi}_{n}^{\text {interior. }}$. The continuous analog of this case has been studied extensively in the literature, in connection to Lindley's equation. Here $U(s) \equiv 0$, $V(s) \equiv 1$, and

$$
F(s)=\frac{H_{\text {in }}(1)}{H_{\text {in }}(s)}, \quad|s| \leqslant 1 .
$$

Case 2. $\boldsymbol{\xi}_{n}^{\text {boundary }} \stackrel{D}{=} \boldsymbol{\xi}_{n}^{\text {interior }}+1$. This case is relevant in $M / G / 1$-type queueing models, including the first variant of our queue with negative customers, see section 3 . Here $U(s) \equiv V(s) \equiv 1$ and

$$
F(s)=H_{\text {in }}(1)\left[\frac{s}{H_{\text {in }}(s)}+C \cdot(s-1)\right], \quad|s| \leqslant 1 .
$$


We now define the matrix $M$ and the vector $\vec{\vartheta}$. Their dimension is

$$
d \triangleq \max \{m, l+1\}+1 \text {. }
$$

First,

$$
M \triangleq\left[\begin{array}{ccccc|cccc}
1 & 0 & 0 & \ldots & 0 & 0 & 0 & \ldots & 0 \\
\hline 1 & \omega_{1} & \omega_{1}^{2} & \ldots & \omega_{1}^{m} & \omega_{1}^{m+1} & \omega_{1}^{m+2} & \ldots & \omega_{1}^{\ell+1} \\
1 & \omega_{2} & \omega_{2}^{2} & \ldots & \omega_{2}^{m} & \omega_{2}^{m+1} & \omega_{2}^{m+2} & \ldots & \omega_{2}^{\ell+1} \\
\vdots & \vdots & \vdots & & \vdots & \vdots & \vdots & & \vdots \\
1 & \omega_{m} & \omega_{m}^{2} & \ldots & \omega_{m}^{m} & \omega_{m}^{m+1} & \omega_{m}^{m+2} & \ldots & \omega_{m}^{\ell+1} \\
\hline 0 & 0 & 0 & \ldots & 0 & 1 & 0 & \ldots & 0 \\
0 & 0 & 0 & \ldots & 0 & 0 & 1 & \ldots & 0 \\
\vdots & \vdots & \vdots & & \vdots & \vdots & \vdots & \ddots & \vdots \\
0 & 0 & 0 & \ldots & 0 & 0 & 0 & \ldots & 1
\end{array}\right]
$$

The determinant of $M$ is equal to that of the upper left $(m+1) \times(m+1)$ block. This block is the transpose of a Vandermonde matrix (see, e.g., [12, p. 35]), whose determinant is

$$
\prod_{0 \leqslant i<j \leqslant m}\left(\omega_{j}-\omega_{i}\right)
$$

with $\omega_{0} \triangleq 0$. Hence, $M$ is nonsingular under the hypothesis of the theorem. sentation

For defining $\vec{\vartheta}$ we need the first coefficients $\zeta_{0}, \ldots, \zeta_{\ell-m}$ of the Laurent repre-

$$
H_{\text {out }}(s) / s=\sum_{i=0}^{\infty} \zeta_{i} s^{-i}
$$

Such a representation exists, by equation (4). The $\zeta_{i}$ are easily calculated as the Taylor coefficients $(1 / i !) J^{(i)}(0)$ of the function

$$
J(s) \triangleq s H_{\text {out }}(1 / s)=\frac{\prod_{\left\{j /\left|\beta_{j}\right|<1\right\}}\left(1-s \beta_{j}\right)}{\prod_{\left\{i /\left|\alpha_{i}\right|<1\right\}}\left(1-s \alpha_{i}\right)} .
$$

We can now define $\vec{\vartheta}$ via

$$
\vartheta_{i} \triangleq \begin{cases}\left(v_{0}-u_{0}\right) H_{\text {in }}(0), & i=0, \\ \left(\omega_{i}-1\right) U\left(\omega_{i}\right) H_{\text {in }}\left(\omega_{i}\right), & i=1, \ldots, q, \\ -C^{-1} U\left(\omega_{i}\right) H_{\text {out }}\left(\omega_{i}\right), & i=q+1, \ldots, m, \\ -C^{-1} \sum_{j=0}^{\ell-i+1} u_{i+j-1} \zeta_{j}, & i=m+1, \ldots, \ell+1 .\end{cases}
$$

Note that the indexing of $\vec{\vartheta}$ starts from zero, and that the values substituted in $H_{\text {in }}(s)$ and $H_{\text {out }}(s)$ are in the proper domains. 
Remark 2.2. As written in equation (9), the expression for $F(s)$ is generally not in a reduced form, because $V(s)$ may have zeros in $|s| \leqslant 1$ while $F(s)$ cannot have any poles there. The factor $\prod_{i=1}^{q}\left(s-\omega_{i}\right)$ must cancel out. To see that every $\omega_{i}, 1 \leqslant i \leqslant q$, is also a zero of the term in brackets, observe that $\vec{s} M^{-1} \vec{\vartheta}$ with $s=\omega_{i}$ is equal to the $i$ th element of $M M^{-1} \vec{\vartheta}$, namely to $\vec{\vartheta}_{i}$.

\section{The queueing model at embedded epochs}

\subsection{The first variant}

Consider a classical $\mathrm{M} / \mathrm{G} / 1$ queue with an arrival rate $\lambda$, and with a service distribution function $S(t)$ which has mean $b$, second moment $b^{(2)}$, and a rational Laplace-Stieltjes transform $B(z)$. Augment the system with the following feature: In addition to the normal "positive" customers, there is also an independent Poissonian stream, of rate $\nu$, of "negative" customers; those remove work from the system, rather than add work to it. In this paper we assume the following removal mechanism. Upon the $n$th service completion, the numbers $w_{n}^{+}$and $w_{n}^{-}$of positive and negative waiting customers are compared ${ }^{3}$. When $\boldsymbol{w}_{n}^{-} \leqslant w_{n}^{+}$, every negative customer removes one positive customer. Otherwise $\left(\boldsymbol{w}_{n}^{-}>\boldsymbol{w}_{n}^{+}\right)$, only the available $\boldsymbol{w}_{n}^{+}$positive customers can be removed. In either case the system is then cleared of negative customers. A new service commences immediately, if a positive customer is available. The number $\boldsymbol{x}_{n}$ of positive customers left in the system immediately after the $n$th service completion is thus

$$
\boldsymbol{x}_{n}=\left[\boldsymbol{w}_{n}^{+}-\boldsymbol{w}_{n}^{-}\right]^{+} .
$$

Let $\boldsymbol{a}_{n}^{+}$and $\boldsymbol{a}_{n}^{-}$denote the numbers of positive and negative customers arriving during the first service which occurs after the $n$th service completion. Consider the evolution of the chain $\left\{\boldsymbol{x}_{n}\right\}$. When $\boldsymbol{x}_{n-1}>0$, a new service begins immediately after the $(n-1)$ th service completion. Hence

$$
\left.\begin{array}{l}
\boldsymbol{w}_{n}^{+}=\boldsymbol{x}_{n-1}+\boldsymbol{a}_{n-1}^{+}-1 \\
\boldsymbol{w}_{n}^{-}=\boldsymbol{a}_{n-1}^{-}
\end{array}\right\} \quad \text { if } \boldsymbol{x}_{n-1}>0
$$

When $x_{n-1}=0$, the $(n-1)$ th service completion is followed by an idle period. In the variant considered here, we assume that negative customers arriving during an idle period leave immediately. The first positive customer who triggers the next service period is not included in $\boldsymbol{a}_{n}^{+}$, and must be counted separately. Hence

$$
\left.\begin{array}{l}
\boldsymbol{w}_{n}^{+}=\boldsymbol{x}_{n-1}+\boldsymbol{a}_{n-1}^{+} \\
\boldsymbol{w}_{n}^{-}=\boldsymbol{a}_{n-1}^{-}
\end{array}\right\} \quad \text { if } \boldsymbol{x}_{n-1}=0
$$

${ }^{3}$ The "+" and "-." superscripts should not be confused with the []$^{+}$and []$^{-}$operators. 
The chain $\left\{\boldsymbol{x}_{n}\right\}$ is thus a random walk of the type described in the previous section, with

$$
\xi_{n}^{\text {interior }}=a_{n}^{+}-a_{n}^{-}-1,
$$

and corresponds to the second special case of Remark 2.1. Note that $a_{n}^{+}-a_{n}^{-}-1$ is not bounded either from below or from above. The stability condition (2) translates into $\lambda-\nu<1 / b$. For obtaining the steady-state generating function of $\left\{\boldsymbol{x}_{n}\right\}$ we need an expression for $A(s)$. Conditioning on the length of the service period and observing that $\boldsymbol{a}_{n}^{+}$and $\boldsymbol{a}_{n}^{-}$are conditionally independent, we obtain

$$
\begin{aligned}
A(s) & =s^{-1} \mathbb{E}\left(s^{a_{n}^{+}-a_{n}^{-}}\right)=s^{-1} \int_{t=0}^{\infty} \mathrm{e}^{-\lambda t(1-s)} \mathrm{e}^{-\nu t\left(1-s^{-1}\right)} \mathrm{d} S(t) \\
& =s^{-1} B\left(\lambda(1-s)+\nu\left(1-s^{-1}\right)\right) .
\end{aligned}
$$

To summarize, we list the procedure for computing the stationary generating function of the number of positive customers at service completion epochs.

1. Determine $A(s)$ using equation (18).

2. Find the representation (3).

3. Obtain the requested generating function from equation (11).

Example 3.1 (M/M/1 queue with negative customers). Assume that the service is exponential, with rate $1 / b=\mu>\lambda-\nu$. The service transform is then

$$
B(z)=\mu /(\mu+z)
$$

Hence

$$
A(s)-1=s^{-1} \frac{\mu}{\mu+\left[\lambda(1-s)+\nu\left(1-s^{-1}\right)\right]}-1=\frac{\lambda s^{2}-(\lambda+\nu+\mu) s+\nu+\mu}{-\lambda s^{2}+(\lambda+\nu+\mu) s-\nu} .
$$

This function must have a zero at $s=1$, and have the form

$$
C \frac{(s-1)\left(s-\alpha_{1}\right)}{\left(s-\beta_{1}\right)\left(s-\beta_{2}\right)} \text {. }
$$

Solving for the constants yields

$$
C=-1, \quad \alpha_{1}=\rho^{-1}, \quad \beta_{1,2}=\frac{1}{2}\left(1+\rho^{-1} \mp \kappa\right),
$$

where

$$
\rho \triangleq \frac{\lambda}{\nu+\mu}, \quad \kappa \triangleq \sqrt{\left(1+\rho^{-1}\right)^{2}-4 \frac{\nu}{\lambda}}
$$


$\alpha_{1}$ is outside the unit circle. This fact alone is sufficient to imply, by equation (4), that $\beta_{1}$ and $\beta_{2}$ must be real and $\beta_{1}<1<\beta_{2}$. Applying equation (11), we have

$$
F(s)=\frac{1-\alpha_{1}}{1-\beta_{2}}\left[(-1)(s-1)+s \frac{s-\beta_{2}}{s-\alpha_{1}}\right],
$$

which can be written in the form

$$
F(s)=\gamma+(1-\gamma) \frac{1-\rho}{1-s \rho}
$$

with

$$
\gamma=\left(\rho^{-1}-1\right) \frac{\rho^{-1}-(\kappa-1)}{\rho^{-1}+(\kappa-1)} .
$$

We recognize that the inverse of $F(s)$ is a combination of a geometrical distribution with parameter $\rho$ and a point mass $\gamma$ at zero:

$$
\mathbb{P}\left\{\boldsymbol{x}_{n}=i\right\}=\gamma 1_{\{i=0\}}+(1-\gamma)(1-\rho) \rho^{i}, \quad i=0,1, \ldots .
$$

When $\nu=0, \gamma$ is zero and $\mathbb{P}\left\{\boldsymbol{x}_{n}=i\right\}$ reduces to the familiar $\mathrm{M} / \mathrm{M} / 1$ expression $(1-\rho) \rho^{i}$, with $\rho=\lambda / \mu$.

Example 3.2 (M/E $\mathrm{E}_{k} / 1$ queue with negative customers). Assume that the service is Erlang- $k$, with mean $1 / \mu$ such that $\mu>\lambda-\nu$. The service transform is then

$$
B(z)=\left(\frac{k \mu}{k \mu+z}\right)^{k}
$$

Hence

$$
A(s)=\frac{(k \mu)^{k} s^{k-1}}{[s k \mu+\lambda s(1-s)+\nu(s-1)]^{k}} .
$$

An easy calculation shows that the quadratic term between square brackets in the denominator of equation (23) has two real roots, $s_{-}<1<s_{+}$. An alternative way of seeing this is to observe that $A(s)$, being the generating function of a random variable $\xi_{n}^{\text {boundary }}$ which takes both positive and negative values, cannot be analytic throughout the unit disc or throughout its complement. Equations (3) and (4) now imply that

$$
A(s)-1=(1-s) \frac{\prod_{i=1}^{k-1}\left(s-\alpha_{i}^{-}\right) \prod_{i=1}^{k}\left(s-\alpha_{i}^{+}\right)}{\left(s-s_{+}\right)^{k}\left(s-s_{-}\right)^{k}},
$$

with $\left|\alpha_{i}^{-}\right|<1,\left|\alpha_{i}^{+}\right|>1$. Applying equation (11), we have

$$
F(s)=\frac{\prod_{i=1}^{k}\left(1-\alpha_{i}^{+}\right)}{\left(1-s_{+}\right)^{k}}\left[(-1)(s-1)+s \frac{\left(s-s_{+}\right)^{k}}{\prod_{i=1}^{k}\left(s-\alpha_{i}^{+}\right)}\right] .
$$

One can easily verify that $k=1$ yields the results of the previous example. 


\subsection{The second variant}

In this variant, negative customers arriving during an idle period stay in the system until the end of the next service completion. The distribution of the $\boldsymbol{\xi}_{n}^{\text {interior }}$ is unaltered. But at the boundary we have now

$$
\boldsymbol{\xi}_{n}^{\text {boundary }} \stackrel{D}{=} \boldsymbol{\xi}_{n}^{\text {interior }}+1-\boldsymbol{\imath},
$$

where $\imath$ has the same distribution as the number of negative arrivals during an idle period, and is independent of the $\boldsymbol{\xi}_{n}^{\text {interior }}$. Noting that the length of the idle period is distributed exponentially with parameter $\lambda$, we obtain

$$
\frac{A_{0}(s)}{A(s)}-1=s \mathbb{E}\left[\left(\frac{1}{s}\right)^{\imath}\right]-1=s \frac{\lambda}{\lambda+\nu\left(1-\frac{1}{s}\right)}-1=(s-1) \frac{\frac{\lambda}{\lambda+\nu} s-\frac{\nu}{\lambda+\nu}}{s-\frac{\nu}{\lambda+\nu}} .
$$

Theorem 1 is applicable, because $A_{0}(s) / A(s)$ has only one pole, different from zero. To apply the theorem we need

$$
M=\left[\begin{array}{ccc}
1 & 0 & 0 \\
1 & \frac{\nu}{\lambda+\nu} & \left(\frac{\nu}{\lambda+\nu}\right)^{2} \\
0 & 0 & 1
\end{array}\right], \quad \vec{\vartheta}=\left[\begin{array}{c}
0 \\
\frac{\lambda \nu^{2}}{(\lambda+\nu)^{3}} H_{\text {in }}\left(\frac{\nu}{\lambda+\nu}\right) \\
-C^{-1} \frac{\lambda}{\lambda+\nu}
\end{array}\right] .
$$

The inverse of $M$ is

$$
M^{-1}=\left[\begin{array}{ccc}
1 & 0 & 0 \\
-\frac{\lambda+\nu}{\nu} & \frac{\lambda+\nu}{\nu} & -\frac{\nu}{\lambda+\nu} \\
0 & 0 & 1
\end{array}\right]
$$

The computational procedure of the previous subsection remains valid, with only one modification. Instead of equation (11) use equation (9), with $U(s)$ and $V(s)$ taken as the numerator and denominator of the right hand side of equation (26), respectively, and with

$$
\vec{s} M^{-1} \vec{\vartheta}=\frac{\lambda}{\lambda+\nu}\left\{s \frac{\nu}{\lambda+\nu}\left[H_{\text {in }}\left(\frac{\nu}{\lambda+\nu}\right)+C^{-1}\right]-s^{2} C^{-1}\right\} .
$$

Example 3.3 (the $M / M / 1$ queue with negative customers revisited). Consider the model of Example 3.1 with the assumption of the second variant. The new $F(s)$ is obtained by following the modification in the procedure. $F(s)$ corresponds again to a combination of a geometrical distribution and a point mass at zero. The parameter of the geometrical distribution is the same $\rho$, but the point mass at zero is different, and given by

$$
\gamma^{\prime}=\frac{\kappa+\rho^{-1}+1}{\kappa+\rho^{-1}-1} \cdot \frac{2\left(\rho^{-1}-1\right)}{(\kappa+1) \frac{\lambda}{\nu}-\frac{\mu}{\nu}-1-2 \rho^{-1}} .
$$




\section{The arbitrary-time queue-length distribution}

In the previous section it has been shown how one can obtain the generating function $F(s)$ of the queue-length distribution immediately after service completion epochs in the $M / G / 1$ queue with negative customers. In the conventional M/G/1 queue, that distribution coincides with the arbitrary-time queue-length distribution, but that is not the case when negative customers are allowed to remove ordinary customers at service completion epochs. Below we relate the two queue-length distributions for that model.

Let $\Pi(s)$ denote the steady-state generating function of the continuous-time queue-length process $\boldsymbol{x}(t)$. We exploit the fact that, during a service, the queue-length process evolves like a birth process with rate $\lambda$. The number of arrivals (of ordinary customers) during one service has generating function $K(s)=B(\lambda(1-s))$. It is well known that the generating function of the queue-length growth at an arbitrary epoch during a service, since its beginning, equals

$$
\frac{1-K(s)}{\lambda b(1-s)}
$$

Distinguish between the three cases that the system is observed during an idle period, during the first service after an idle period and during a service that immediately follows another service; then we can write

$$
\begin{aligned}
\Pi(s)= & \frac{F(0) / \lambda}{F(0)[1 / \lambda+b]+[1-F(0)] b} \\
& +\frac{F(0) b}{F(0)[1 / \lambda+b]+[1-F(0)] b} \cdot s \cdot \frac{1-K(s)}{\lambda b(1-s)} \\
& +\frac{[1-F(0)] b}{F(0)[1 / \lambda+b]+[1-F(0)] b} \cdot \frac{F(s)-F(0)}{1-F(0)} \cdot \frac{1-K(s)}{\lambda b(1-s)}, \quad|s| \leqslant 1 .
\end{aligned}
$$

Note that the first quotient in each of the three terms gives the probability of each of the above-mentioned three events. Rearranging we find:

$$
\Pi(s)=\frac{F(0)}{F(0)+\lambda b}\left[B(\lambda(1-s))+\frac{F(s)}{F(0)} \cdot \frac{1-B(\lambda(1-s))}{1-s}\right], \quad|s| \leqslant 1 .
$$

Thus $F(s)$ is converted into $\Pi(s)$. Denoting the mean steady-state queue length at an arbitrary time by $\mathbb{E} \boldsymbol{x}(t)=\Pi^{\prime}(1)$, and the mean steady-state queue length immediately after service completions and the consecutive customer killings by $\mathbb{E} \boldsymbol{x}_{n}=F^{\prime}(1)$, it follows from (27) that

$$
\mathbb{E} \boldsymbol{x}(t)=\frac{F(0)}{F(0)+\lambda b}\left[\lambda b\left(1+\frac{\mathbb{E} \boldsymbol{x}_{n}}{F(0)}\right)+\frac{\lambda^{2} b^{(2)}}{2 F(0)}\right] .
$$


Example 4.1 (exponential service). The arbitrary-time distribution obtained from the $B(z)$ and $F(s)$ of equations $(19,20)$ is

$$
\mathbb{P}\{\boldsymbol{x}(t)=i\}=\theta(1-\sigma) \sigma^{i}+(1-\theta)(1-\rho) \rho^{i}, \quad i=0,1, \ldots,
$$

where $\rho$ is the same as before, $\sigma=\lambda /(\lambda+\mu)$, and

$$
\theta=\frac{\gamma+\left(1-\rho^{-1}\right) \frac{\nu}{\mu}}{\sigma\left(1-\frac{\nu}{\lambda}\right)\left(\gamma+\rho^{-1}+\frac{\nu}{\mu}\right)} .
$$

Remark 4.1. In a conventional M/G/1 queue, a level crossing argument and the PASTA principle imply that $F(s)=\Pi(s)$. Substitution in equation (27) gives

$$
F(s)=\frac{(1-\rho)(1-s) B(\lambda(1-s))}{B(\lambda(1-s))-s} .
$$

This is the well-known Pollaczek-Khintchine formula, which thus finds an alternative proof.

\section{Proof of the random walk result and discussion of alternative derivations}

\subsection{Proof of Theorem 1}

Define

$$
\boldsymbol{Y}_{n} \triangleq\left[\boldsymbol{X}_{n-1}+\boldsymbol{\xi}_{n-1}\right]^{-}, \quad n=0, \pm 1, \ldots
$$

and

$$
G(s) \triangleq \mathbb{E}\left(s^{Y_{n}}\right)-1, \quad|s| \geqslant 1 .
$$

These objects serve as proof mainstays, by enabling the elimination of the []$^{+}$operator from the evolution equation (1). This equation indeed becomes

$$
\boldsymbol{X}_{n+1}+\boldsymbol{Y}_{n+1}=\boldsymbol{X}_{n}+\boldsymbol{\xi}_{n}
$$

We express the generating functions of the two sides in terms of $A(s), A_{0}(s), F(s)$, and $G(s)$. First,

$$
\mathbb{E}\left(s^{X_{n+1}+Y_{n+1}}\right)=F(s)+G(s), \quad|s|=1,
$$

simply because $s^{x+y}=s^{x}+s^{y}-1$ holds when $x y=0$. Second,

$$
\begin{aligned}
\mathbb{E}\left(s^{\boldsymbol{X}_{n}} s^{\boldsymbol{\xi}_{n}}\right)= & \mathbb{E}\left(s^{\boldsymbol{X}_{n}} s^{\boldsymbol{\xi}_{n}} \mid \boldsymbol{X}_{n}=0\right) \mathbb{P}\left(\boldsymbol{X}_{n}=0\right) \\
& +\mathbb{E}\left(s^{\boldsymbol{\xi}_{n}} \mid \boldsymbol{X}_{n}>0\right) \mathbb{E}\left(s^{\boldsymbol{X}_{n}} \mid \boldsymbol{X}_{n}>0\right) \mathbb{P}\left(\boldsymbol{X}_{n}>0\right) \\
= & A_{0}(s) F(0)+A(s)[F(s)-F(0)], \quad|s|=1 .
\end{aligned}
$$


Equating the two expressions yields

$$
F(s)[A(s)-1]=G(s)-F(0)\left[A_{0}(s)-A(s)\right], \quad|s|=1 .
$$

This single functional equation determines the two unknowns $F(s)$ and $G(s)$. It can be solved in several ways, depending on the preferred representation of the data and the solution. We solve for $F(s)$ using the data described in subsection 2.1, and discuss other derivations in the next subsection. Putting the representations $(5,8)$ in the equation, we obtain

$$
\begin{aligned}
& H_{\text {in }}(s)[F(s) V(s)+(s-1) F(0) U(s)] \\
& \quad=C^{-1} H_{\text {out }}(s)\left[\frac{G(s)}{s-1} V(s)-F(0) U(s)\right], \quad|s|=1 ;
\end{aligned}
$$

note that $s=1$ is a zero of $G(s)$. The last equation is the basis for applying the standard Wiener-Hopf arguments. The left and right hand sides are analytical in the domains $|s| \leqslant 1$ and $|s| \geqslant 1$, respectively. Hence there exists some entire function $\psi(s)$ with which they coincide in these domains. The right hand side is $o\left(s^{d}\right)$ as $s \rightarrow \infty$. According to Liouville's theorem, $\psi(s)$ must be a polynomial of a degree not exceeding $d-1$. Write this polynomial as $\vec{s} \cdot \vec{a}$, where $\vec{a}=\left[a_{0}, \ldots, a_{d-1}\right]$ is the $d$-vector of coefficients. $F(s)$ can now be extracted from the left hand side of equation (32),

$$
F(s)=\frac{1}{V(s)}\left[\frac{\vec{s} \cdot \vec{a}}{H_{\text {in }}(s)}-(s-1) F(0) U(s)\right], \quad|s| \leqslant 1,
$$

but the constants $\vec{a}$ and $F(0)$ need to be resolved. We validate hereafter that

$$
M \vec{a}=F(0) \vec{\vartheta}
$$

so $\vec{a}=F(0) M^{-1} \vec{\vartheta}$. Putting $s=1$ in the left hand side of equation (32) gives

$$
H_{\text {in }}(1) V(1)=\overrightarrow{1} \cdot \vec{a}=F(0) \overrightarrow{1} M^{-1} \vec{\vartheta} .
$$

It was noted before that both $H_{\text {in }}(s)$ and $V(s)$ cannot have $s=1$ as a zero. Also, $F(0)=\mathbb{P}\left\{\boldsymbol{X}_{n}=0\right\} \neq 0$, since the random walk is positive recurrent. This proves that $\overrightarrow{1} M^{-1} \vec{\vartheta} \neq 0$. The required constants are thus given by

$$
F(0)=\frac{H_{\text {in }}(1) V(1)}{\overrightarrow{1} M^{-1} \vec{\vartheta}}, \quad \vec{a}=\frac{H_{\text {in }}(1) V(1)}{\overrightarrow{1} M^{-1} \vec{\vartheta}} M^{-1} \vec{\vartheta}
$$

It remains to validate equation (34). The validation is done by blocks, corresponding to those appearing in equation (15). Equating the left hand side of equation (32) to the polynomial $\vec{s} \cdot \vec{a}$, and putting $s=0$ and $s=\omega_{1}, \ldots, \omega_{q}$ gives the first two blocks. 
A similar use of the right hand side of equation (32) with $s=\omega_{q+1}, \ldots, \omega_{m}$ gives the third block ${ }^{4}$. The fourth block is validated by considering the behavior of the right hand side of equation (32) as $s \rightarrow \infty$. We rewrite it using equation (14), and equate it again to $\vec{s} \cdot \vec{a}$ on $|s| \geqslant 1$ :

$$
\underbrace{C^{-1} \frac{H_{\text {out }}(s)}{s} G(s) \frac{s}{s-1} V(s)}_{\triangleq f_{1}(s)}-\underbrace{F(0) C^{-1} s\left(\sum_{j=0}^{\ell} u_{j} s^{j}\right)\left(\sum_{i=0}^{\infty} \frac{\zeta_{i}}{s^{i}}\right)}_{\triangleq f_{2}(s)}=\sum_{j=0}^{\max \{m, l+1\}} a_{j} s^{j} .
$$

Observe that $f_{1}(s)=o\left(s^{m+1}\right)$, so $a_{m+1}, \ldots, a_{\ell+1}$ are determined solely by $f_{2}(s)$. We identify these coefficients as the convolutions appearing at the fourth entry of equation (15). Equation (34) is established.

\subsection{Alternative assumptions and derivations}

\subsubsection{Extension to the case were the hypothesis of Theorem I is not fulfilled}

The derivation of the previous subsection can be extended to the case where $\omega_{1}, \ldots, \omega_{m}$ are not distinct from each other or from zero. However, $M$ and $\vec{\vartheta}$ take a more involved and less regular form. Suppose that $\omega$ is a zero of the polynomial $V(s)$, of multiplicity $p$. We exploit the fact that $\omega$ is also a zero of the derivatives $V^{(i)}(s), i=1, \ldots, p-1$. If $|\omega| \leqslant 1$, we put $s=\omega$ in each of the corresponding derivatives of the equation that equates the left hand side of equation (32) to $\vec{s} \cdot \vec{a}$. If $\omega=0$, we also use the derivative of order $p$. If $\left|\omega_{i}\right|>1$, we use the right hand side of equation (32) instead of the left hand side. Thus $p$ linear equations in $F(0)$ and $\vec{a}$ are produced, fully compensating against the loss due to the multiplicity. Previously, the lines of $M$ were distinct substitutions in the vector of powers $\vec{s}$. Now, some lines of $M$ may constitute substitutions in its derivatives $\left(\mathrm{d}^{j} / \mathrm{d} s^{j}\right) \vec{s}$. The lines of $M$ are of the following form:

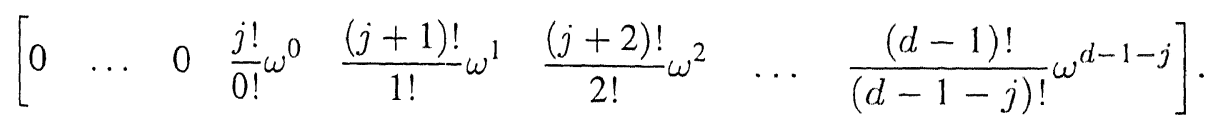

Every two lines differ either in the value substituted for $s$, or in the order $j$ of the derivative. It is readily seen that a square matrix formed according to this rule is nonsingular (e.g., when $\omega_{1}=\omega_{2}$, the determininant of the modified matrix $M$ is equal to the partial derivative w.r.t. $\omega_{2}$ of the determinant of the original matrix given in equation (13), at the point $\omega_{1}=\omega_{2}$. The explicit formula for the Vandermonde determinant shows that this derivative is non-zero). Hence, the extended system determines $F(s)$ uniquely.

\footnotetext{
4 In partitioning the zeros of $V(s)$, the assignment of those on the unit circle to the second block rather than to the third was arbitrary. If $\omega_{i}$ is on the unit circle, it must be a zero of $A(s)$ (see equation (8)), so it must satisfy $\left(\omega_{i}-1\right) H_{\text {in }}\left(\omega_{i}\right)=-C^{-1} H_{\text {out }}\left(\omega_{i}\right)$, by equation (5).
} 


\subsubsection{Using a direct representation of the boundary generating function}

There always exists a reduced representation

$$
A_{0}(s)-1=(s-1) \frac{W_{\text {out }}(s)}{\prod_{i=1}^{y}\left(s-\eta_{i}\right)}, \quad|s|=1,
$$

where $W_{\text {out }}(s)$ is a rational function, analytical in $|s| \geqslant 1$, and $\eta_{1}, \ldots, \eta_{y}$ lie in $|s|>1$. Putting the representations $(5,35)$ instead of $(5,8)$ in equation $(31)$, we obtain

$$
\begin{aligned}
& H_{\text {in }}(s)[F(s)-F(0)] \prod_{i=1}^{y}\left(s-\eta_{i}\right) \\
& \quad=C^{-1} H_{\text {out }}(s)\left[\frac{G(s)}{s-1} \prod_{i=1}^{y}\left(s-\eta_{i}\right)-F(0) W_{\text {out }}(s)\right], \quad|s|=1 .
\end{aligned}
$$

The extraction of $F(s)$ from this equation is analogous to its extraction from equation (32). As before, there exists a polynomial which coincides with the two sides at the appropriate domains. To extract its coefficients and $F(0)$, we put $s=0$ at the left hand side and $s=\eta_{1}, \ldots, \eta_{y}$ at the right hand side. We also analyze the behavior of the right hand side as $s \rightarrow \infty$, if the degrees involved require that, using the Laurent expansions of $H_{\text {out }}(s) / s$ and $W_{\text {out }}(s)$. If $\eta_{1}, \ldots, \eta_{y}$ are not all distinct, we revert to differentiation, as discussed in the previous paragraph.

\subsubsection{Representation through projections}

Boxma and Lotov [5] give a representation of the solution $F(s)$ of equation (31) through $[0, \infty)$-projections; the $\mathcal{D}$-projection of a series $\sum_{i=-\infty}^{\infty} a_{i} s^{i}$ is defined to be $\sum_{i \in \mathcal{D}} a_{i} s^{i}$.

\section{Acknowledgements}

We are pleased to acknowledge the comments of Professor J.W. Cohen (CWI) and of Dr. V.I. Lotov from the Institute of Mathematics at Novosibirsk.

\section{References}

[1] S. Asmussen, Applied Probability and Queues (Wiley, New York, 1975).

[2] N. Bayer, On the identification of Wiener-Hopf factors, Queueing Systems, this issue.

[3] A.A. Borovkov, Stochastic Processes in Queueing Theory (Springer-Verlag, Berlin, 1976).

[4] R.J. Boucherie and O.J. Boxma, The workload in the M/G/1 queue with work removal, Probability in the Engineering and Informational Sciences 10 (1996) 261-277. 
[5] O.J. Boxma and V.I. Lotov, On a class of one-dimensional random walks, Markov Processes and Related Fields 2 (1996) 349-362.

[6] J.W. Cohen, The Wiener-Hopf technique in applied probability, in: Perspectives in Probability and Statistics, ed. J. Gani (Applied Probability Trust, 1975) pp. 145-156.

[7] J.W. Cohen, The Single Server Queue (North-Holland, Amsterdam, 1982).

[8] J.W. Cohen and O.J. Boxma, Boundary Value Problems in Queueing System Analysis (NorthHolland, Amsterdam, 1983).

[9] E. Gelenbe, Product-form queueing networks with negative and positive customers, Journal of Applied Probability 28 (1991) 656-663.

[10] P.G. Harrison and E. Pitel, The M/G/1 queue with negative customers, Advances in Applied Probability 28 (1996) 540-566.

[11] L. Kleinrock, Queueing Systems, Volume I: Theory (Wiley, New York, 1975).

[12] P. Lancaster and M. Tismenetsky, The Theory of Matrices (Academic Press, New York, second edition, 1985). 